\title{
MÖSSBAUER STUDIES OF IRON-BASED SUPERCONDUCTORS
}

\author{
ARTUR BŁACHOWSKI ${ }^{1}$, ALEKSANDRA K. JASEK ${ }^{1}$, KAMILA KOMĘDERA ${ }^{1}$, ANGELIKA PIERZGA ${ }^{1}$, \\ KRZYSZTOF RUEBENBAUER ${ }^{1} \& \mathrm{JAN}^{\mathrm{Z} U K R O W S K I^{2}}$ \\ ${ }^{1}$ Mössbauer Spectroscopy Division, Institute of Physics, Pedagogical University, Poland \\ ${ }^{2}$ Academic Centre for Materials and Nanotechnology, AGH University of Science and Technology, Poland
}

\begin{abstract}
This contribution is a review concerned with the microscopic characterization of complex materials by using transmission Mössbauer spectroscopy - mainly $14.4-\mathrm{keV}$ resonant transition in ${ }^{57} \mathrm{Fe}$. Attention is focused on the novel superconductors, i.e. iron-based superconductors, which are extensively investigated in our Mössbauer laboratory, primarily versus sample temperature. Iron-based superconductors make four major families based on the corrugated nearly-two-dimensional sheets of either strongly bound iron-pnictogen or iron-chalcogen atoms. Usually, superconductivity is induced by doping or applying pressure to the parent compound, except the simplest compounds of the ' 11 ' family. One can dope any kind of atom within the compound in isovalent, hole-doping or electrondoping fashion. Parent compounds exhibit itinerant magnetic order of the $3 \mathrm{~d}$ (iron) character. It appears as spin density wave (SDW) of the antiferromagnetic type incommensurates with the respective lattice period and of the complex shape. For a majority of cases, it is a longitudinal SDW propagating along the $a$-axis of the orthorhombic unit cell being created at the magnetic order from the tetragonal cell due to the magneto-elastic forces. On the other hand, the $3 \mathrm{~d}$ magnetism and orthorhombic distortion are gone for superconductors as shown by the Mössbauer spectra obtained versus temperature, and by spectra obtained in the strong external magnetic field at low temperatures - stronger than the first critical field for these second kind superconductors. However, superconductivity is intimately related to these layered structures with the electronic charge modulation, leading to the charge density wave (CDW) on iron nuclei - observed as variation of the isomer shift. What is more, one observes closely related modulation of the electric field gradient on iron nuclei called electric field gradient wave (EFGW). The shape of these modulations changes rapidly at the superconducting gap: opening and relaxing back once the bosonic system of Cooper pairs is well separated from the rest of the electronic system. It was found that localized $4 \mathrm{f}$ magnetic moments order within the superconducting phase in a similar fashion as in the normal phase.
\end{abstract}

Keywords: iron-based superconductors, Mössbauer spectroscopy, charge density wave, electric field gradient wave.

\section{INTRODUCTION}

Usually $3 \mathrm{~d}$ magnetic order and superconductivity are mutually exclusive within the same electronic system - particularly ferromagnetic type of order and s-type superconductivity. However, diamagnetic forms of iron exist and are able to form stable compounds. The majority of these compounds have large energy gap and respective compounds are insulators or semi-conductors at best [1]. Hence, they cannot exhibit superconductivity. A small group of iron compounds is simultaneously metallic and diamagnetic as far as $3 \mathrm{~d}$ magnetism is considered. Hence, they might become superconductors at low temperatures. Even pure iron in the hexagonal $\varepsilon$-form exhibits superconducting island, albeit upon having applied hydrostatic pressure [2]. On the other hand, it is easily magnetically polarized in the strong external magnetic field. A discovery of layered iron-based unconventional superconductors in 2008 by the Hosono team was a real breakthrough [3]. They have many similarities to cuprates [4], albeit exhibit many differences, as the parent compounds are metallic and exhibit itinerant $3 \mathrm{~d}$ antiferromagnetism. The common feature for all iron-based 
superconductor families is presence of strongly coupled iron-pnictogen or iron-chalcogen layers with tetrahedral iron coordination within the layer. Hence, the iron occurs nominally in the divalent state [5]-[7]. There are four major families of these compounds excluding some more complex derivatives. All of them crystallize in the tetragonal symmetry, and parent compounds undergo orthorhombic distortion closely related to the $3 \mathrm{~d}$ magnetic order. The simplest is ' 11 ' family being just a stack of FeSe layers. This compound has no parent and exhibits superconductivity below about $8 \mathrm{~K}$ under normal pressure, albeit orthorhombic distortion occurs at much higher temperature [8]. The ' 111 ' family differs by intercalation of iron-chalcogen layers by some diamagnetic ions like lithium [9]. Much higher superconducting transition temperatures could be obtained for ' 111 ' and ' 1111 ' families. The parent compound of the '111' family has composition $\mathrm{AFe}_{2} \mathrm{As}_{2}$ with $\mathrm{A}$ being some metal including magnetic $4 \mathrm{f}$ elements with either almost pure spin magnetic moment like $\mathrm{Eu}^{2+}$ or $\mathrm{Gd}^{3+}$ ions or ions having significant orbital contribution to the magnetic moment. $4 \mathrm{f}$ magnetic moments remain highly localized, if present. Large, almost perfect single crystals of the ' 111 ' family compounds could be grown. It is interesting to note, that two adjacent FeAs layers within ' 111 ' compounds are mutually inverted, while for remaining families simple stacking occurs. The ' 1111 ' family has larger separation between FeAs layers as the separator is made of cation and anion like for example in PrFeAsO. The parent compound could be converted into superconductor by replacement of any element, i.e., by hole, electronic or isovalent doping. One can apply hydrostatic pressure as well [10]. Upon doping $3 \mathrm{~d}$ magnetism diminishes, transition to the orthorhombic phase disappears, and superconductivity occurs. For the majority of cases there is some optimal doping. For overdoped material a transition temperature to the superconducting state lowers and eventually disappear leading to the metallic and 3d diamagnetic state [11], [12]. Larger separation between iron bearing layers leads to higher superconducting transition temperatures due to reduced dimensionality [13]. Macroscopic measurements show some coexistence of the $3 \mathrm{~d}$ magnetic order and superconductivity for underdoped samples.

Resonant transition of 14.4-keV between ground and first excited state of ${ }^{57} \mathrm{Fe}$ is the best Mössbauer transition from the experimental point of view. Natural iron contains about 2.14 at $\%$ of ${ }^{57} \mathrm{Fe}$ and it is a major constituent of the compounds described above. Hence, it seems natural to use transmission Mössbauer spectroscopy to look upon local properties of ironbased superconductors. One can look upon hyperfine interactions of the iron nucleus and get some information about dynamics from the recoilless fraction and second order Doppler shift (SOD). Resonant transition of 21.6-keV connecting ground and first excited state of ${ }^{151} \mathrm{Eu}$ could be used as well for compounds bearing europium as natural europium contains about 47.8 at $\%$ of ${ }^{151} \mathrm{Eu}$.

\section{BASIC FEATURES OF MÖSSBAUER SPECTROSCOPY}

For time independent Mössbauer spectra one can get information on the dynamics of the resonant atom via measured recoilless fraction and SOD. On the other hand, much more information yields hyperfine interactions including isomer shift, electric quadrupole interaction and eventually magnetic dipole interaction. Resolution of the $14.4-\mathrm{keV}$ transition in ${ }^{57} \mathrm{Fe}$ is good enough to derive information on the hyperfine magnetic field modulation, and to some limited extent on the electronic charge distribution on the resonant sites.

\subsection{Hyperfine interactions}

Basically one observes the following hyperfine interactions: 1) the isomer shift due to the finite electron density within resonant nucleus, 2) electric quadrupole interaction, and 3) 
magnetic dipole interaction. The isomer shift is expressed as $\delta=\alpha\left(\rho_{1}-\rho_{2}\right)$ with the parameter $\alpha$ being calibration constant for a given resonant transition, $\rho_{1}$ denoting electron density on the resonant nucleus, and $\rho_{2}$ standing for corresponding density in the adopted standard. For $14.4-\mathrm{keV}$ transition in ${ }^{57} \mathrm{Fe}$ one has $\alpha=-0.291(2) \mathrm{el}^{-1} \mathrm{a} . \mathrm{u}^{3} \mathrm{mms}^{-1}$ and adopted standard is natural metallic $\alpha-\mathrm{Fe}$ at room temperature with about $\rho_{2}=15322.046$ el./a.u. ${ }^{3}$ neglecting thermal expansion [14].

The electric quadrupole interaction is caused by the interaction between nuclear spectroscopic electric quadrupole moment and the electric field gradient on the nucleus. The electric field gradient (EFG) tensor field could be expressed in terms of the stationary electric charge distribution in the following way [15]:

$$
\begin{gathered}
\mathbf{V}(\mathbf{r})=\left(\frac{3}{4 \pi \varepsilon_{0}}\right) \\
\int_{0}^{2 \pi} d \phi \int_{0}^{\pi} d \vartheta \sin \vartheta\left(\begin{array}{ccc}
\sin ^{2} \vartheta \cos ^{2} \phi-1 / 3 & \sin ^{2} \vartheta \sin \phi \cos \phi & \sin \vartheta \cos \vartheta \cos \phi \\
\sin ^{2} \vartheta \sin \phi \cos \phi & \sin ^{2} \vartheta \sin ^{2} \phi-1 / 3 & \sin \vartheta \cos \vartheta \sin \phi \\
\sin \vartheta \cos \vartheta \cos \phi & \sin \vartheta \cos \vartheta \sin \phi & \cos ^{2} \vartheta-1 / 3
\end{array}\right) \int_{R_{0}>0}^{+\infty}\left(\frac{d R}{R}\right) \rho(\mathbf{r}+\mathbf{R})(1) \\
\text { with } \mathbf{R}=R\left(\begin{array}{c}
\sin \vartheta \cos \phi \\
\sin \vartheta \sin \phi \\
\cos \vartheta
\end{array}\right)
\end{gathered}
$$

Here, the symbol $\varepsilon_{0}$ denotes electric permeability of vacuum. Eventual charge of the probing nucleus is excluded from calculations, while the exclusion zone (eventually containing probing nucleus) described by the radius $R_{0}$ contains remaining charge having nearly spherical symmetry (except eventual linear terms do not contributing to the EFG tensor), and hence do not contributing to the EFG, as one needs symmetry lower than cubic (around given point) to have non-zero EFG. The integral is convergent for large distances as the matter is neutral on sufficiently large scales. The symbol $\rho(\mathbf{r}+\mathbf{R})$ denotes total density of the electric charge. One can see that the EFG tensor is symmetric and traceless. Hence, it can be always diagonalized locally and it is invariant upon axes inversion. It has axial symmetry provided a three fold or four fold axis is present at the point considered $\mathbf{r}$. For a local diagonal representation one can write the following expression:

$$
\mathbf{V}=V_{33}\left(\begin{array}{ccc}
-\frac{1}{2}(1-\eta) & 0 & 0 \\
0 & -\frac{1}{2}(1+\eta) & 0 \\
0 & 0 & 1
\end{array}\right)
$$

The so-called asymmetry parameter $\eta=\left(V_{11}-V_{22}\right) / V_{33}$ satisfies the following condition $0 \leq \eta \leq 1$ provided one has $\left|V_{11}\right| \leq\left|V_{22}\right| \leq\left|V_{33}\right|$ and $V_{33} \neq 0$. For axially symmetric EFG one has $\eta=0$. The electric quadrupole part of the hyperfine Hamiltonian takes on the following form in above principal coordinates: 


$$
\begin{aligned}
& \mathbf{H}_{Q}=A_{Q}\left[3 \mathbf{I}_{3}^{2}-\mathbf{I}^{2}+\eta\left(\mathbf{I}_{1}^{2}-\mathbf{I}_{2}^{2}\right)\right] \text { with } A_{Q}=\left(\frac{e Q V_{33}}{4 I(2 I-1)}\right)\left(\frac{c}{E_{0}}\right) \text { or } \\
& \mathbf{H}_{Q}=\left(\frac{e Q}{2 I(2 I-1)}\right)\left(\frac{c}{E_{0}}\right)(\mathbf{I} \bullet \mathbf{V} \bullet \mathbf{I}), \mathbf{I}=\left(\begin{array}{c}
\mathbf{I}_{1} \\
\mathbf{I}_{2} \\
\mathbf{I}_{3}
\end{array}\right) .
\end{aligned}
$$

Here, the symbol $e$ denotes positive elementary charge, the symbol $Q$ stands for spectroscopic nuclear quadrupole moment in a given nuclear state, the symbol $I$ denotes spin of this nuclear state, $C$ stands for speed of light in vacuum, and $E_{0}$ denotes resonant transition energy. The symbols $\mathbf{I}_{1}, \mathbf{I}_{2}, \mathbf{I}_{3}$ denote nuclear spin projection operators on respective Cartesian axes. The symbol $\mathbf{I}^{2}$ stands for $\mathbf{I}^{2}=\mathbf{I}_{1}^{2}+\mathbf{I}_{2}^{2}+\mathbf{I}_{3}^{2}$. One can note that splitting could be observed provided $I>\frac{1}{2}$. Hence, no quadrupole interaction is seen in the ground state of ${ }^{57} \mathrm{Fe}$ with $I_{g}=\frac{1}{2}$, and a doublet is created in the first excited state with $I_{e}=\frac{3}{2}$ - the spectroscopic moment for this state amounts to $Q_{e}=+0.17(1) \mathrm{b}$. The splitting of this state amounts to $\Delta=2|\varepsilon|$ with $\varepsilon=\frac{1}{4} Q_{e} V_{33}\left(c / E_{0}\right) \sqrt{1+\eta^{2} / 3}$.

Magnetic dipole interaction could be described in the point-like approximation for 14.4$\mathrm{keV}$ transition in ${ }^{57} \mathrm{Fe}$. It occurs provided some magnetic order exists unless some external field is applied. The ground state splits into two levels, while the excited into four levels. Usually one sees six lines unless excited state Hamiltonian is highly non-diagonal due to the large and misaligned EFG as the transition is almost pure M1 transition. The occupation of the ground state sub-levels could be assumed as being equal each other unless extremely low temperatures occur. Magnetic part of the hyperfine Hamiltonian could be written in the following form in coordinates defined in previous paragraph:

$$
\mathbf{H}_{M}=-\mu_{N} g B\left(\frac{c}{E_{0}}\right)\left[\mathbf{I}_{3} \cos \beta+\sin \beta\left(\mathbf{I}_{1} \cos \gamma+\mathbf{I}_{2} \sin \gamma\right)\right] \text { or } \mathbf{H}_{M}=-\mu_{N} g\left(\frac{c}{E_{0}}\right)(\mathbf{B} \bullet \mathbf{I}) \text {. }
$$

The symbol $\mu_{N}$ denotes nuclear magneton. The symbol $g$ stands for the gyro-magnetic factor of the given nuclear state, while the symbol $B$ denotes value of the total magnetic field acting on the nucleus. Angles $\beta$ and $\gamma$ stand for the polar and azimuthal angles, respectively, of the hyperfine field axial vector $\mathbf{B}$ in the coordinates used. One has magnetic Hamiltonian solely for the ground state, while for the excited state one obtains $\mathbf{H}=\mathbf{H}_{Q}+\mathbf{H}_{M}+S \mathbf{1}$ with $S$ being a total shift due to the isomer shift and SOD. For small EFG one can write eigenvalues of the excited state in the following approximate form provided the magnetic interaction dominates:

$$
\begin{aligned}
& \left\langle m_{e}^{\prime}|\mathbf{H}| m_{e}\right\rangle= \\
& \delta_{m_{e} m_{e}^{\prime}}\left\{-\mu_{N} g_{e} B\left(\frac{c}{E_{0}}\right) m_{e}+\left(\frac{e Q_{e} V_{33}}{24}\right)\left(\frac{c}{E_{0}}\right)\left[3 \cos ^{2} \beta-1+\eta \sin ^{2} \beta \cos (2 \gamma)\right]\left(3 m_{e}^{2}-\frac{15}{4}\right)+S\right\} .
\end{aligned}
$$

Here the symbols $m_{e}$ and $m_{e}^{\prime}$ denote nuclear magnetic quantum numbers. The electric quadrupole interaction apparently vanishes for the so-called magic angle $\beta \approx 54.74^{\circ}$ within 
this approximation provided $\eta=0$. The sign of the quadrupole coupling constant could be determined in the presence of the magnetic splitting (here practically in the case of axially symmetric EFG). Expression (5) allows to find extremely small electric quadrupole interaction - particularly for $\beta=0$ [16].

\subsection{Modulated structures}

The simplest modulated structure is a $3 \mathrm{~d}$ itinerant magnetism with the magnetic moment (hyperfine field) varying along some crystal direction. Such modulation is called spin density wave (SDW) and it could be longitudinal (moments aligned with the modulation direction), perpendicular or mixed. For the perpendicular case one can expect various forms of fans or spirals. The SDW is quite often incommensurate with the respective lattice period. Hence, various resonant nuclei experience various hyperfine fields. Usually the modulation is some form of interlaced antiferromagnetic structures. Such structures become commensurate provided the number of the space dimensions is suitably increased.

Another form could be atomic positions in quasi-crystals or charge modulation (charge density wave - CDW) leading eventually to the respective modulation of the EFG (electric field gradient wave $-\mathrm{EFGW}$ ). In general, modulated field $\mathbf{A}(\mathbf{r})$ versus position $\mathbf{r}$ could be expanded in the plane waves of the form [15], [17]:

$$
\mathbf{A}(\mathbf{r})=\sum_{k=1}^{K} \sum_{n=1}^{N(K)}\left\{\mathbf{a}_{k n}(\mathbf{r}) \cos \left[n\left(\mathbf{q}_{k} \bullet \mathbf{r}\right)\right]+\mathbf{b}_{k n}(\mathbf{r}) \sin \left[n\left(\mathbf{q}_{k} \bullet \mathbf{r}\right)\right]\right\}: K \geq 1, N(K) \geq 1 .
$$

The index $k$ enumerates various field components, while the index $n$ respective harmonics of these components with the even amplitudes $\mathbf{a}_{k n}(\mathbf{r})$ and eventual corresponding odd components $\mathbf{b}_{k n}(\mathbf{r})$. For the majority of cases one can assume that above amplitudes do not depend on current position $\mathbf{r}$. Each sub-field might have characteristic wave vector. Resonant transition of $14.4-\mathrm{keV}$ in ${ }^{57} \mathrm{Fe}$ is very sensitive to the shape of the SDW. Sinusoidal SDW with small admixture of higher harmonics is observed in chromium, while the original idea of this type of order is due to Overhauser.

In the case of CDW and/or EFGW some approximations are usually needed due to the limited resolution of the method. The EFGW is usually approximated as distribution of the parameter $\varepsilon=\frac{1}{4} Q_{e} V_{33}\left(c / E_{0}\right) \sqrt{1+\eta^{2} / 3}$ in the following form $\varepsilon(\mathbf{q} \bullet \mathbf{r})=\varepsilon_{0}+A F_{\max }^{-1} F(\mathbf{q} \bullet \mathbf{r})$ The parameter $\varepsilon_{0}$ describes eventual constant component. The parameter $A$ denotes amplitude of the modulation, while the parameter $F_{\max }$ is the maximum of the function $F(\mathbf{q} \bullet \mathbf{r})$. The latter function takes on the following form [18]:

$$
F(\mathbf{q} \bullet \mathbf{r})=\sin (\mathbf{q} \bullet \mathbf{r})\left\{\exp \left[-\beta^{2}\left(\frac{\mathbf{q} \bullet \mathbf{r}}{2 \pi}-\frac{1}{4}\right)^{2}\right]+\exp \left[-\beta^{2}\left(\frac{\mathbf{q} \bullet \mathbf{r}}{2 \pi}-\frac{3}{4}\right)^{2}\right]\right\} .
$$

The phase angle covers the range $0 \leq \mathbf{q} \bullet \mathbf{r}<2 \pi$ (quite often range $0 \leq \mathbf{q} \bullet \mathbf{r} \leq \pi$ suffices), while the parameter $\beta$ is responsible for the shape. In the case of CDW one can usually estimate dispersion only by applying expression $\Delta \rho=\sqrt{\left(\Gamma^{2}-\Gamma_{\exp }^{2}\right) / \alpha^{2}}$. Here $\Gamma>\Gamma_{\exp }$ stands for the actual absorber linewidth, while $\Gamma_{\exp }$ is the corresponding linewidth in the 
absence of the modulation. The symbol $\alpha$ is the previously defined calibration constant for the isomer shift, and $\Delta \rho$ is the dispersion of the electron density on resonant nuclei [18].

\section{SELECTED RESULTS}

A commercial ${ }^{57} \mathrm{Co}(\mathrm{Rh})$ sources were used for ${ }^{57} \mathrm{Fe}$ spectroscopy. They were kept at room temperature. All reported shifts are reported against natural $\alpha$-Fe kept at room temperature for ${ }^{57} \mathrm{Fe}$ spectroscopy.

\subsection{Iron selenide}

Essential results for the $\mathrm{Fe}_{1.01} \mathrm{Se}$ compound with the superconducting transition at about $8 \mathrm{~K}$ are summarized below [19]. Spectra exhibit quadrupole split doublets with a small jump in the isomer shift at the tetragonal-orthorhombic transition. This jump corresponds to the lowering of the electron density on the iron nucleus in the Cmma orthorhombic low temperature phase by 0.02 el./a.u. ${ }^{3}$ in comparison with the tetragonal $\mathrm{P} 4 / \mathrm{nmm}$ phase at the same temperature. A transition occurs at about $105 \mathrm{~K}$.

There are no traces of the magnetic order. Hence, the spectra were obtained in the longitudinal external magnetic field and they are shown in Fig. 1. The applied fields exceeded the first critical field at temperatures of measurements. Due to the fact, that only very small volume of the sample retains superconductivity between first and second critical field almost all iron atoms are exposed to the applied field.

Hence, it was proven that the compound is truly diamagnetic and very hard to polarize by the external magnetic field, as the observed field is practically the same as applied field. This finding excludes exotic magnetically based coupling boson fields leading to the formation of the Bose quasi-particles and subsequently to the Bose condensate responsible for superconductivity.

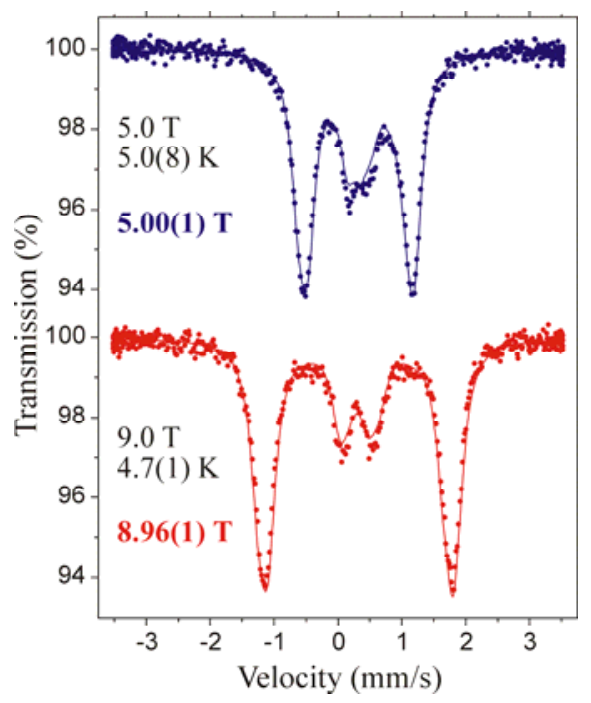

Figure 1: ${ }^{57} \mathrm{Fe}$ iron selenide spectra obtained in the superconducting orthorhombic state and in the longitudinal external field of either 5.0 T and temperature $5.0(8) \mathrm{K}$ or $9.0 \mathrm{~T}$ and $4.7(1) \mathrm{K}$. Corresponding hyperfine fields were obtained as 5.00(1) $\mathrm{T}$ and 8.96(1) T [19]. 


\subsection{CDW and EFGW sensitivity to the superconducting transition}

Optimally doped ' 122 ' compound $\mathrm{Ba}_{0.6} \mathrm{~K}_{0.4} \mathrm{Fe}_{2} \mathrm{As}_{2}$ does not exhibit any magnetic order. The superconducting gap for this compound begins to open at $38 \mathrm{~K}$ and the gap is fully formed by $24 \mathrm{~K}$ provided there is neither external magnetic field nor hydrostatic pressure. It is worth noting that the superconductor is obtained by the hole doping of the parent compound $\mathrm{BaFe}_{2} \mathrm{As}_{2}$ [18]. On the other hand, the ' 1111 ' superconductor $\mathrm{SmFeAsO}{ }_{0.91} \mathrm{~F}_{0.09}$ is obtained from the parent $\mathrm{SmFeAsO}$ by electron doping. The gap for the latter superconductor starts to open at $47 \mathrm{~K}$ and the gap formation is completed by $38 \mathrm{~K}$. Unfortunately, some traces of SDW appear for the latter case, but fortunately at about $20 \mathrm{~K}$ and below [20]. Fig. 2 shows ${ }^{57} \mathrm{Fe}$ spectra of the $\mathrm{Ba}_{0.6} \mathrm{~K}_{0.4} \mathrm{Fe}_{2} \mathrm{As}_{2}$ compound collected around transition to the superconducting state (left panel), while the corresponding spectra of the $\mathrm{SmFeAsO}_{0.91} \mathrm{~F}_{0.09}$ compound are shown in the right panel of Fig. 2.

One can clearly see change of the spectrum shape at the gap opening and partial recovery upon full development of the gap. Changes have opposite character for the hole and electron doped sample.

Fig. 3 shows evolution of the EFGW across superconducting transition and complete formation of the Bose condensate with corresponding variations in the electron density $\Delta \rho$ on the iron nuclei versus temperature.

It is evident that changes have opposite signs depending on the addition to or removal of electrons from the system.

\section{CONCLUSIONS}

It is clear that superconducting and $3 \mathrm{~d}$ magnetically ordered electronic systems are mutually exclusive, and no $3 \mathrm{~d}$ intrinsic magnetic moments exist within superconductor. On the other hand, one has to allow for tunneling of the Bose condensate across magnetic regions and vice versa, as the $3 \mathrm{~d}$ magnetism is of the itinerant character. Hence, it seems unlikely that magnetic excitations of any kind generate bosonic field responsible for coupling electrons into pairs.

Superconductors obtained by hole or electron doping from otherwise magnetic metallic material exhibiting itinerant $3 \mathrm{~d}$ magnetism with significant magneto-elastic forces develop CDW and EFGW instead of structural transition. The latter charge waves are sensitive to the superconducting gap opening and complete formation. Changes are opposite for hole and electron doping.
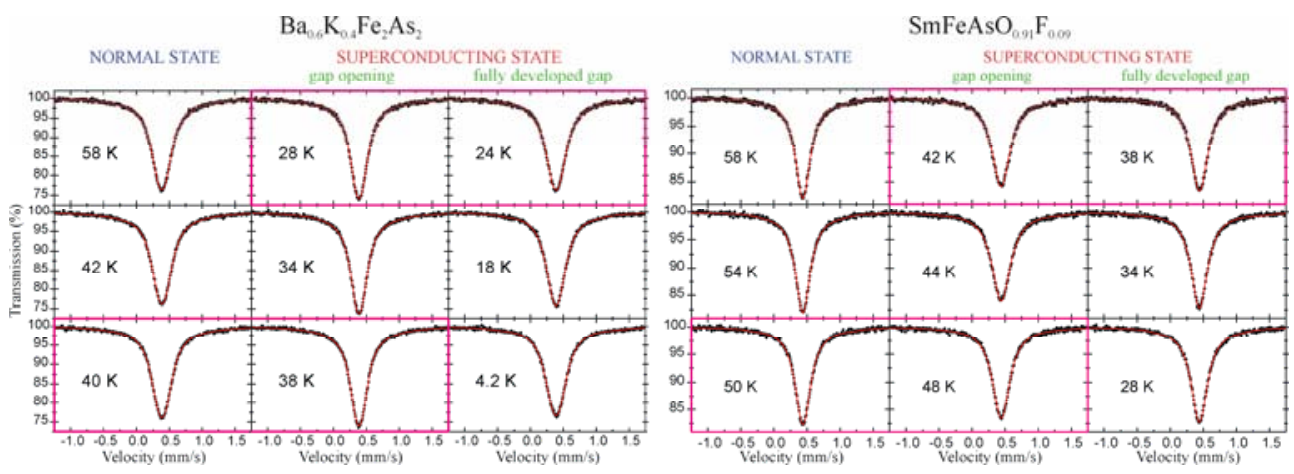

Figure 2: $\quad{ }^{57} \mathrm{Fe}$ spectra for the optimally doped $\mathrm{Ba}_{0.6} \mathrm{~K}_{0.4} \mathrm{Fe}_{2} \mathrm{As}_{2}$ compound around transition to the superconducting state (left panel) [18]. Right panel shows similar spectra of the $\mathrm{SmFeAsO} \mathrm{O}_{0.91} \mathrm{~F}_{0.09}$ superconductor around transition to the superconducting state [20]. 

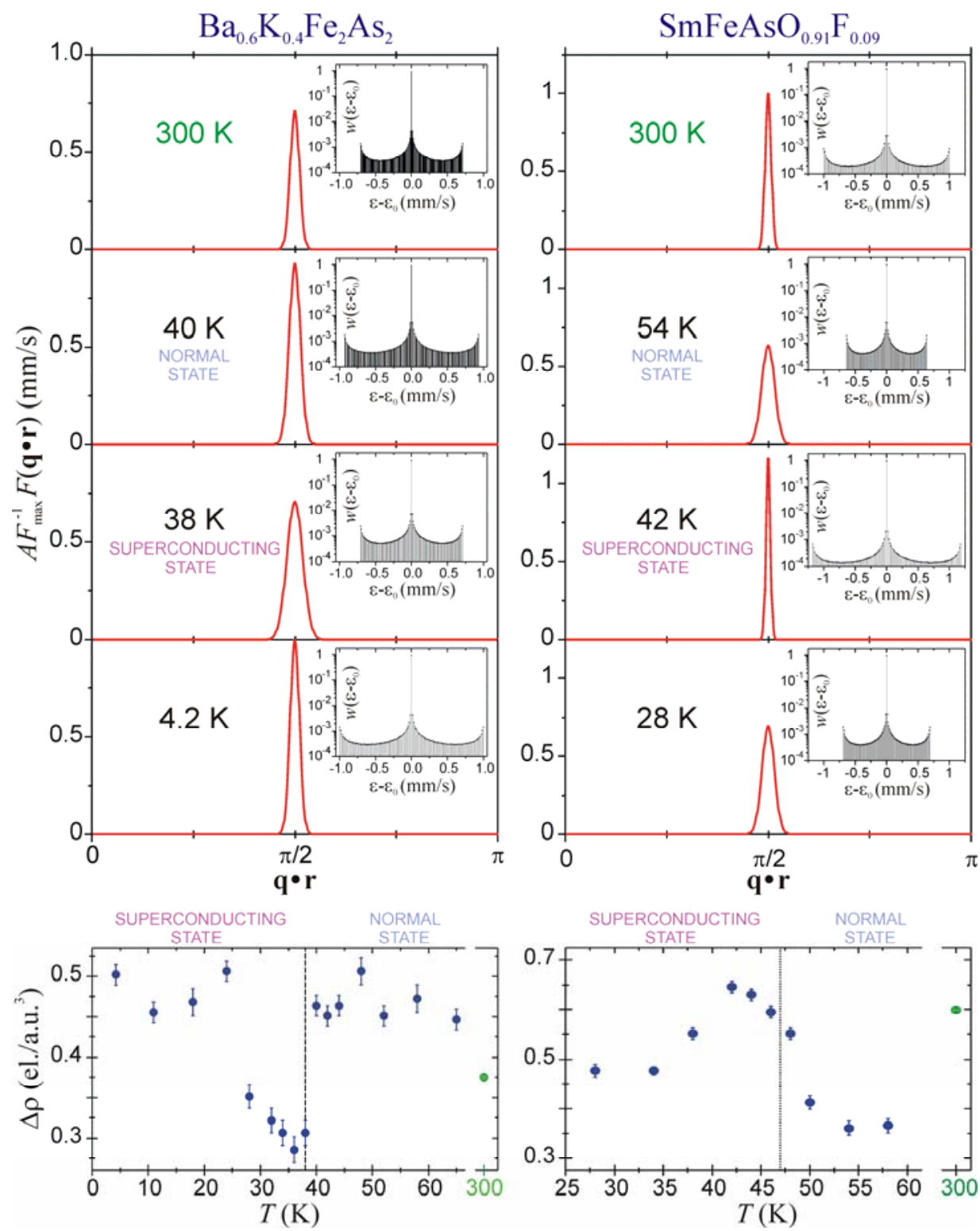

Figure 3: Shape of the EFGW for selected temperatures versus phase $\mathbf{q} \bullet \mathbf{r}$. Insets show corresponding normalized weights $w\left(\varepsilon-\varepsilon_{0}\right)$ of the quadrupole coupling constant $\varepsilon-\varepsilon_{0}$. Left panel shows EFGW for $\mathrm{Ba}_{0.6} \mathrm{~K}_{0.4} \mathrm{Fe}_{2} \mathrm{As}_{2}$, while the right panel EFGW for $\mathrm{SmFeAsO}_{0.91} \mathrm{~F}_{0.09}$. For the latter case, one has $\varepsilon_{0}=0$. Lower part shows variation of the electron density $\Delta \rho$ on the iron nuclei during formation of the Bose condensate. Left panel shows results for $\mathrm{Ba}_{0.6} \mathrm{~K}_{0.4} \mathrm{Fe}_{2} \mathrm{As}_{2}$ [18], while the right panel for $\mathrm{SmFeAsO}_{0.91} \mathrm{~F}_{0.09}$ [20]. Dashed vertical lines mark superconducting transition temperature. 


\section{REFERENCES}

[1] Hazony, Y., Effect of Zero Point Motion on the Mössbauer Spectra of $\mathrm{K}_{4} \mathrm{Fe}(\mathrm{CN})_{6}$ and $\mathrm{K}_{4} \mathrm{Fe}(\mathrm{CN})_{6} \cdot 3 \mathrm{H}_{2} \mathrm{O}$. J. Chem. Phys., 45(7), pp. 2664-2668, 1966.

[2] Katsuya, S., Tomohiro, K., Shigeyuki, F., Keiki, T., Kazuyoshi, K., Yoshichika, O. \& Kiichi, A., Superconductivity in the non-magnetic state of iron under pressure. Nature, 412, pp. 316-318, 2001.

[3] Yoichi, K., Takumi, W., Masahiro, H. \& Hidei, H., Iron-Based Layered Superconductor $\mathrm{La}\left[\mathrm{O}_{1-\mathrm{x}} \mathrm{F}_{\mathrm{x}}\right] \mathrm{FeAs}(\mathrm{x}=0.05-0.12)$ with $\mathrm{T}_{\mathrm{c}}=26 \mathrm{~K} . \mathrm{J}$. Am. Chem. Soc., 130(11), pp. 3296-3297, 2008.

[4] Bednorz, J.G. \& Müller, K.A., Possible high $\mathrm{T}_{\mathrm{c}}$ superconductivity in the $\mathrm{Ba}-\mathrm{La}-\mathrm{Cu}-\mathrm{O}$ system. Z. Phys. B, 64(2), pp 189-193, 1986.

[5] Toshihide, T., Howe Arthur, T. \& Greenwood Norman, N., The $\mathrm{Fe}_{1+x} \mathrm{Se}$ system. I. Mössbauer spectra and electrical conductivity of $\mathrm{Fe}_{1.04} \mathrm{Se}$. J. Solid State Chem., 17(12), pp. 157-163, 1976.

[6] Jain, B.K, Singh, A.K. \& Chandra, K., An investigation of binary system Fe-Se. J. Phys. F: Metal Phys., 8(12), pp. 2625-2630, 1978.

[7] Reddy, K.V. \& Chetty, S.C., Mössbauer Studies on the Fe-Se System. Phys. Stat. Sol. (a), 32(2), pp. 585-592, 1975.

[8] McQueen, T.M. et al., Extreme sensitivity of superconductivity to stoichiometry in $\mathrm{Fe}_{1+\delta}$ Se. Phys. Rev. B, 79, 014522, 2009.

[9] Błachowski, A., Ruebenbauer, K., Żukrowski, J., Przewoźnik, J. \& Marzec, J., Mössbauer and magnetic measurements of superconducting LiFeP. J. Alloys Compd., 505(2), pp. L35-L37, 2010.

[10] Kitao, S. et al., Spin Ordering in LaFeAsO and Its Suppression in Superconductor $\mathrm{LaFeAsO}_{0.89} \mathrm{~F}_{0.11}$ Probed by Mössbauer Spectroscopy. J. Phys. Soc. Jpn., 77, 103706, 2008.

[11] Błachowski, A., Ruebenbauer, K., Żukrowski, J., Rogacki, K., Bukowski, Z. \& Karpinski, J., Shape of spin density wave versus temperature in $\mathrm{AFe}_{2} \mathrm{As}_{2}(\mathrm{~A}=\mathrm{Ca}, \mathrm{Ba}$, Eu): A Mössbauer study. Phys. Rev. B, 83, 134410, 2011.

[12] Błachowski, A. et al., Interplay between magnetism and superconductivity in EuFe 2 ${ }_{\mathrm{x}} \mathrm{Co}_{\mathrm{x}} \mathrm{As}_{2}$ studied by ${ }^{57} \mathrm{Fe}$ and ${ }^{151} \mathrm{Eu}$ Mössbauer spectroscopy. Phys. Rev. B, 84, 174503, 2011.

[13] Komędera, K., et al. Structural disorder in $\mathrm{Li}_{\mathrm{x}}\left(\mathrm{C}_{5} \mathrm{H}_{5} \mathrm{~N}\right)_{\mathrm{y}} \mathrm{Fe}_{2-\mathrm{z}} \mathrm{Se}_{2}$ and $\mathrm{Cs}_{\mathrm{x}} \mathrm{Fe}_{2-\mathrm{z}} \mathrm{Se}_{2}$ superconductors studied by Mössbauer spectroscopy. J. Magn. Magn. Mater., 406, pp. 244-250, 2016.

[14] Wdowik, U.D. \& Ruebenbauer, K., Calibration of the isomer shift for the $14.4-\mathrm{keV}$ transition in ${ }^{57} \mathrm{Fe}$ using the full-potential linearized augmented plane-wave method. Phys. Rev. B, 76, 155118, 2007.

[15] Jasek, A.K., Charge density waves in iron-based superconductors studied by Mössbauer Spectroscopy, Mössbauer Spectroscopy Division, Institute of Physics, Pedagogical University, Ph.D. Thesis (supervisor: Błachowski A.): Cracow, 2016 (in Polish).

[16] Błachowski, A., Komędera, K., Ruebenbauer, K., Cios, G., Żukrowski, J, \& Górnicki, R., Electric quadrupole interaction in cubic BCC $\alpha$-Fe. J. Alloys Compd., 673, pp. 420425, 2016.

[17] Ruebenbauer, K. \& Duraj, Ł., www.elektron.up.krakow.pl/mosgraf-2009.

[18] Jasek, A.K. et al., Electric field gradient wave (EFGW) in iron-based superconductor $\mathrm{Ba}_{0.6} \mathrm{~K}_{0.4} \mathrm{Fe}_{2} \mathrm{As}_{2}$ studied by Mössbauer spectroscopy. J. Alloys Compd., 609, pp. 150$155,2014$. 
[19] Błachowski, A., Ruebenbauer, K., Żukrowski, J., Przewoźnik, J., Wojciechowski, K. \& Stadnik, Z.M., Mössbauer spectroscopy evidence for the lack of iron magnetic moment in superconducting FeSe. J. Alloys Compd., 494(1-2), pp. 1-4, 2010.

[20] Jasek, A.K. et al., Change of the charge modulation during superconducting transition in $\mathrm{SmFeAsO}_{0.91} \mathrm{~F}_{0.09}$ seen by ${ }^{57} \mathrm{Fe}$ Mössbauer spectroscopy. J. Alloys Compd., 658, pp. 520-524, 2016. 